\title{
CONSIDERACIONES ESENCIALES SOBRE EL TEMA ÉTICO EN LA INVESTIGACIÓN EDUCATIVA
}

\section{ESSENTIAL CONSIDERATIONS ON THE ETHICAL ISSUE IN EDUCATIONAL RESEARCH}

\author{
OSVALDO HERNÁNDEZ GONZÁLEZ*, ANTONIO ANTONIO**, \\ DANIELA GONZÁLEZ FERNÁNDEZ***, MILTON CONTRERAS SÁEZ****
}

\section{RESUMEN}

La ética es un campo complejo que ha ido cobrando mucha fuerza en el último tiempo en la investigación educativa, por su relevancia para garantizar la confianza, la dignidad, la privacidad, la confidencialidad y el anonimato de quienes participan en ella. Este artículo busca poner de relieve algunas consideraciones necesarias sobre los principales dilemas éticos que se presentan en la investigación educativa para estar a la altura del desarrollo social contemporáneo.

Palabras clave: comité de ética, investigación educativa, ciencias de la educación.

\section{ABSTRACT}

Ethics is a complex field that has gained a lot of strength in the last five years of educational research, due to its relevance to trust in the trust, dignity, privacy, confidentiality and anonymity of those who participate in it. This article seeks to highlight some necessary opinions about the main ethical dilemmas that arise in educational research to live up to contemporary social development.

Key words: ethics committee, educational research, educational science.

\footnotetext{
* Magíster en educación Especial y Psicopedagogía. Estudiante del Doctorado en Ciencias Humanas, Universidad de Talca, Talca, Chile. osvaldo.hernandez@utalca.cl

** Magíster en Educación Especial y Psicopedagogía. Estudiante del Doctorado en Educación, Universidad del Estado de Santa Catarina, Florianópolis, Brasil. antoniotonny1988@hotmail. com

*** Magíster en Gestión y Políticas Educativas. Facultad de Ciencias de la Salud. Universidad Autónoma de Chile, Talca, Chile. dgonzalezf@uautonoma.cl

**** Magíster en Estudios y Desarrollo de la Familia. Facultad de Ciencias Sociales y Humanidades. Universidad Autónoma de Chile, Talca, Chile. milton.contreras@uautonoma.cl
} 


\section{Introducción}

La Ética es una rama de la filosofía que tiene como propósito fundamental reflexionar críticamente sobre aspectos relacionados a lo moral en la vida social y en la conducta humana (González, González \& Ruiz, 2012).

En las sociedades contemporáneas, es plausible la utilización de los aspectos vinculados a la ética en el campo educativo como plataforma idónea para potenciar la búsqueda de vías que propicien cambios profundos en los sistemas de enseñanza, en el progreso humano y en el desarrollo de los vínculos sociales.

A la luz de los criterios de González, González \& Ruiz (2012), "la ética aplicada a la educación es aquella que aporta los fundamentos teóricos, metodológicos y normativos sobre la moral y los valores para alcanzar la educación integral de la personalidad" (p. 1). En este sentido, se puede afirmar que la aplicación de los principios éticos funciona como una brújula de orientación teóricapráctica para actuar con rectitud y justicia en la construcción de una exploración científica en el campo de las Ciencias de la Educación, como estrategia de enseñanza transversal.

A pesar de que históricamente en múltiples áreas del conocimiento, se han ido generando códigos éticos (ver la actividad de las Asociaciones Americanas de Sociología, Antropología y Psicología, el Código de Núremberg, la Declaración de Helsinki) para asegurar y vigilar las buenas prácticas en la actividad científica, es necesario subrayar que la investigación educativa no ha recibido las mismas cuotas de interés. Resulta necesario destacar los aportes realizados por la American Educational Research Association a finales del siglo XX, al desarrollar los Ethical Standards of the American Educational Research Association, en los que se hace alusión directa al aspecto ético en el campo de investigación (González, González \& Ruiz, 2012, Moreno, 2011).

En el último tiempo es posible notar, en el campo de las ciencias educativas, un creciente interés por los códigos éticos en la investigación, constituyéndose una temática de alta consideración a la hora de producir resultados científicos que tengan como base la protección de los participantes y la honestidad científica (González, González \& Ruiz, 2012).

Por tales razones, el objetivo del presente trabajo es discutir la importancia de la ética en la investigación educativa contemporánea, haciendo énfasis en su desarrollo, específicamente en los principios, problemas y mecanismos de evaluación que regulan la ética en dicho campo. 


\section{El papel de la Ética en la Investigación Educativa}

La investigación en todas sus modalidades constituye un acto complejo que está orientado a la creación y ampliación del conocimiento, a la solución y resolución de problemas prácticos (Manterola \& Otzen, 2013). En este sentido, la aplicación y ejecución del proceder investigativo adquiere características específicas de índole ética al vincularse con el campo educacional, puesto que la acción científica está mediada y enriquecida por otros procesos complejos relacionados con los intercambios sociales.

Según los argumentos de Buendía \& Berrocal (2001), la investigación educativa es aquella que emerge de la práctica educativa y que, a su vez, la trasforma positivamente. En la misma línea, Palamidessi, Gorostiaga \& Suasnábar (2014), apuntan que:

la investigación educativa se desarrolla en contextos institucionales diversos y en función de una compleja y multiforme vinculación, no sólo en el campo del saber académico de las Ciencias sociales y las Humanidades, sino también en el terreno de los saberes técnicoburocráticos y de las prácticas pedagógicas (p. 1).

Desde esta perspectiva, podemos apreciar que la investigación educativa constituye un campo heterogéneo que se caracteriza por el desarrollo de actividades intensas y duraderas que involucran la actuación de diversos agentes sociales, involucrando la capacidad creadora y transformadora del ser humano, en las cuales la comunicación y la moral tienen una importancia vital.

Ahora bien, a la hora de desarrollar una actividad de esta categoría, los investigadores responsables de conducir el proceso de indagación científica no deben preocuparse solamente por alcanzar logros que estén mediados por su capacidad de producir cambios reales en la práctica o por aumentar y ensanchar considerablemente el conocimiento vinculado con el quehacer educativo, sino que también deben vigilar con sumo cuidado las cuestiones éticas que emergen en su desarrollo, respetando el contexto en el cual se encuentran.

En este sentido, se coincide con los criterios de Sikes, Nixon \& Carr (2003), en que "la investigación educativa está cimentada, epistemológicamente, en los fundamentos morales de la práctica educativa. Son sus propósitos epistemológicos y morales los que subrayan la utilidad y la relevancia de la investigación educativa que importa" (p. 22). Así, el carácter dialéctico de las 
problemáticas educativas requiere de una visión holística que no solo subraye la utilidad y la relevancia de los conocimientos sino también los aspectos morales que la componen.

La dimensiónética en la investigación educativala condicionayla determina de manera exponencial, ya que ésta, a diferencia de otras investigaciones influye notoriamente en la estabilidad y seguridad de los sujetos. Asimismo, la supremacía del paradigma cualitativo en la investigación educativa conforma un complejo marco de relaciones interpersonales en el cual se enfatiza el papel de la ética como plataforma para guiar las intenciones y los intereses específicos del investigador (Badilla, 2006, Ministerio de Educación, 2018).

Sañudo (2006) destaca que "el investigador educativo debeestar consciente de las responsabilidades que adquiere en función de los problemas que va a investigar, de las posibles consecuencias del proceso y de los medios que se eligen para lograr los propósitos" (p. 2). No obstante, dichas responsabilidades se entrelazan constantemente con el quehacer praxeológico en contextos diferenciadores que no necesariamente convergen con la intencionalidad del investigador.

\section{Problemas y principios éticos de la investigación educativa}

Con la intención de dirigir sobre la base de principios fundamentales (honestidad, integridad, probidad o veracidad) un proceso de indagación científica productivo, a lo largo de los últimos años es plausible la participación de códigos éticos y patrones conductuales en la investigación educativa que estimulan, orientan y potencian considerablemente el respeto por los derechos humanos, por las condiciones de intimidad y la transparencia en el proceso de indagación científica.

Es necesario subrayar que el desarrollo histórico de estas formas de actuación en el campo de la bioética (Díaz \& Segado, 2016) han servido como marco de referencia para las investigaciones desarrolladas en educación.

Este cruce interdisciplinar nos ayuda a comprender que

los problemas éticos pueden analizarse a la luz de los que considera los tres principios básicos de la ética aplicada a la investigación: el principio de respeto por las personas, el principio de beneficencia y el principio de justicia; los cuales aplican a través de tres 
procedimientos: razón riesgo-beneficio, consentimiento informado y selección equitativa de los sujetos (Barrio \& Simón, 2006, p. 1).

Estas acciones metodológicas proporcionan las bases esenciales para cumplir a cabalidad estos principios que cumplen con un papel rector dentro de la realización del proceso investigativo y pueden ser aplicados perfectamente en proyectos abordados desde cualquier paradigma.

A la luz de los criterios de Buendía \& Berrocal (2001), en el ámbito de la investigación educativa mayormente se citan cuatro problemas éticos fundamentales: "ocultar a los participantes la naturaleza de la investigación; exponer a los participantes a actos que podrían perjudicarles o disminuir su propia estimación; invadir la intimidad de los participantes y privarlos de los beneficios" (p. 32).

Otra propuesta sobre los dilemas éticos fue planteada por Hammersley \& Traianou (2012). Estos autores mencionan en primer lugar, que se debe minimizar el daño, no solo a los participantes de la investigación, sino que a otros investigadores. Segundo, el respeto de la autonomía, donde a los participantes se les debe permitir decidir si participar o no. Tercero, mencionan la protección a la privacidad, es decir, cómo se mantienen en confidencialidad los datos, considerando que la investigación tiene como objetivo hacerlos públicos. Cuarto, la reciprocidad de oferta, es decir, qué pueden esperar los participantes a cambio de colaborar en la investigación. Y finalmente, la equidad en el trato a las personas, es decir, que ningún participante debe ser injustamente discriminado o favorecido.

En la investigación educativa, el investigador debe utilizar estos principios y además estar alerta para no contradecirlosy, por sobre todo, no incumplirlos. Esto último es un gran desafío, debido a la variabilidad de interpretaciones que tienen los investigadores según su área de experticia.

En aras de proporcionar una visión más amplia de las múltiples problemáticas de carácter ético y de los principios que abarcan la investigación educativa, se vuelve interesante describir analíticamente los criterios planteados por Buendía \& Berrocal (2001), quienes en su artículo La ética de la investigación educativa, señalan tres grandes áreas fundamentales de los problemas éticos: problemas éticos respecto a los participantes, problemas éticos en el desarrollo del trabajo y problemas éticos del propio investigador.

En relación a los participantes, la forma idónea para protegerlos en el transcurso del proceso investigativo es respetando a cabalidad su autonomía, 
informándoles detalladamente cuáles son las intenciones y las motivaciones esenciales del proyecto de investigación al que pudieran estar sujetos. Al principio fundamental de la autonomía se superpone el de la privacidad que exige ocultar sistemáticamente la historia íntima de los que participan y confidencialidad por parte de los que conducen el proceso de indagación científica, si no hubo anonimato al facilitar la información. El respeto a la autonomía y a la privacidad de los participantes constituye pasos de obligatoria presencia para la construcción de una investigación educativa justa y loable moralmente.

Es necesario señalar que, al interior de las investigaciones educativas realizadas bajo el paradigma interpretativo, se desarrollan de manera particular un grupo de principios, destacando "la paridad y la reciprocidad entre todos los participantes involucrados en el proceso investigativo y la protección de la privacidad y la cautela en la emisión de juicios" (Buendía \& Berrocal, 2001, p. 8). En ese sentido, el principio de paridad sienta las bases esenciales para generar condiciones de igualdad y relevancia en las relaciones que se producen entre todos los participantes durante el transcurso de la investigación.

De esta forma, todos los intereses y motivaciones de los participantes son considerados parte sustancial del proceso y no son concebidos como simples medios para lograr determinados propósitos. La privacidad es una cuestión extremadamente relevante porque resguarda la intimidad de los sujetos estudiados. Por su parte, ser moderado y prudente en la emisión de juicios, establece con claridad los límites que no deben ser superados en la descripción de las situaciones que han sido investigadas.

Este análisis, se puede complementar con una interesante discusión considerando que, en ocasiones, el participante principal de la investigación en educación es un niño o un adolescente que, en algunos casos, además pueda tener discapacidad. Diversos autores se han referido a este tema, planteando que la investigación educativa y con niños es necesaria para el desarrollo de las prácticas clínicas (Gabaldón, 2012), y que los aspectos éticos deben ser considerados en la metodología y ser congruentes con las necesidades del niño (Birbeck \& Drummond, 2007). En tal sentido, el Ministerio de Educación (2018), realiza una profunda revisión del tema y entrega orientaciones éticas para las investigaciones en educación con población infantil y /o juvenil, por lo que es de suma importancia considerarlos. 
En cuanto al desarrollo del trabajo, es necesario dimensionar que los problemas éticos pueden abarcar la planificación que sustenta el desarrollo de la investigación y también el espacio que compromete las instancias creadas en aras de socializar los resultados científicos. Ejemplos claros pueden ser: las intenciones inadecuadas que subyacen a las motivaciones fundamentales del investigador (provecho político, publicidad, provecho personal, relaciones públicas, prestigio, justificación de resultados); la toma de decisiones que pueden perturbar la claridad y la transparencia del proceso (prorrogar decisiones críticas, trabajar con muestras intencionales para fines políticos o personales, utilizar influencias para sabotear la investigación); una mala manipulación de los resultados obtenidos (aceptar hipótesis que son falsas, cambiar conclusiones, tergiversar los resultados y utilizarlos de manera que no respondan directamente a los objetivos planteados por la investigación) (González, González \& Ruiz, 2012).

Vigilar cuidadosamente la calidad moral de los cimientos que permiten abordar un problema científico en la esfera educacional, y a su vez dirigir y socializar intencionadamente a través de las vías correctas los resultados obtenidos, constituye una obligación ineludible por parte de los científicos. De no ser así, las maniobras inadecuadas pudieran generar daños intrínsecos y extrínsecos difíciles de reparar por la carga nociva que desembocan para el pleno desarrollo científico y social.

Uno de los problemas éticos que ha levantado mayor expectativa en la comunidad científica es el uso del plagio como resultado del endeble arsenal táctico y la escuálida rectitud moral de algunos autores para enfrentar los embates del proceso investigativo y los retos de su escritura a la hora socializar los resultados. Anderson \& Steneck (2011) plantean que el plagio "consiste en reproducir ideas y palabras de otros autores, haciéndolas pasar por propias y originales, sin el debido reconocimiento o atribución" (p. 739).

Por otra parte, Buendía \& Berrocal (2001), afirman que el acto de plagiar responde mayormente a una de estas categorías: "a) copiar literalmente un trabajo de investigación de otros colegas y presentarlo como propio, b) utilizar trozos de textos o citas de otros autores sin citarlos y c) usar la propiedad intelectual de un autor, sin su permiso expreso" (p. 10).

A través de estudios realizados se ha podido corroborar que el plagio se encuentra entre los tres problemas éticos que se observan como más frecuentes o habituales en los círculos oficiales de divulgación del 
conocimiento científico Latinoamericano (los otros dos son la falta de rigor científico y el fraude) (Domínguez \& Macías, 2004).

Existe cierto consenso en la literatura analizada sobre cuáles son las fuentes principales que estimulan y originan esta conducta desviada. No hay dudas que el insondable crecimiento de los recursos tecnológicos, aparejado con la accesibilidad a grandes volúmenes de información y a las prácticas facilistas aprendidas en el ámbito educativo a lo largo del proceso de formación de la personalidad, ha propiciado y estimulado la aparición de estas formas inmorales de actuación investigativa (Miranda, 2013).

A nuestro juicio, combatir el plagio y sentar las bases para erradicar mayoritariamente la preferencia por estas conductas que disminuyen y demeritan la imagen moral del investigador y de las instituciones a las que responde, debe constituirse como una de las principales preocupaciones de la comunidad científica.

Podemos entender con justa dimensión que son plurales los principios y los problemas éticos que demeritan y entorpecen la Investigación Educativa. Por ello, aquellas investigaciones que se caractericen por sustentar su actividad sobre la base de principios éticos estarán dotadas moralmente para aportar conocimiento con responsabilidad al desarrollo de las Ciencias de la Educación.

\section{Mecanismos de evaluación que regulan la Ética en la Investigación Educativa}

Si asumimos que las prácticas evaluativas al interior de la educación se sustentan principalmente sobre la base de las relaciones humanas, las pautas éticas internacionales para la investigación biomédica en seres humanos dictadas por Organización Panamericana de la Salud y Consejo de Organizaciones Internacionales de las Ciencias Médicas (2016), son un material de consulta obligatoria que se constituye como una brújula orientadora para desarrollar vínculos sociales que promuevan la justicia, el respeto y la protección de los derechos fundamentales de las personas.

En este sentido, Emanuel (1999) y Rodríguez (2004) subrayan el valor, la validez científica, la selección equitativa del sujeto, la proporción favorable de riesgo-beneficio, la evaluación independiente, el consentimiento informado y el respeto por los sujetos inscritos como requisitos cardinales para ayudar a evaluar la ética de las propuestas de investigación que involucran a seres 
humanos. Este conjunto de requisitos actúa y se transforman directamente como guías multidimensionales que nos permiten comprender que cuando se trata de seres humanos es necesario:

- explicitar la importancia científico-social de una investigación y evaluar cuidadosamente que la intervención conlleve a transformaciones positivas para el desarrollo humano sin necesidad de exponerlo a daños que comprometan su bienestar personal y social.

- comprobar la justificación, originalidad y significación científico-social del estudio, así como también las potencialidades teórico-metodológicas de los profesionales que lo llevan a cabo.

- seleccionar los participantes intencional y cuidadosamente, de tal forma que respondan a los intereses particulares de la exploración científica y, a la vez, maximicen los resultados positivos de la investigación en el plano social y de la ciencia.

- reducir los riesgos potenciales a los participantes y agencias de socialización donde se desarrolla, lo cual trae consigo una ampliación de beneficios que impactan en lo personal y lo colectivo.

- efectuar una evaluación independiente en aras de evitar conflictos de intereses, ya que la responsabilidad es social y debe sustentarse sobre reglas y normas de carácter legal.

- contar con los participantes a través de un consentimiento y asentimiento según corresponda, e informarle a cabalidad de las motivaciones fundamentales de las investigaciones en aras de resguardar su autonomía y de empoderarlos con las posibles consecuencias que se derivan de los estudios (Comisión Nacional de Investigación Científica y Tecnológica, 2008).

Este último punto merece especial atención, puesto que el consentimiento informado "es un proceso ético-práctico imprescindible en la operacionalización de estudios científicos: tiene un sustento ético y legal conocido por quienes realizan tareas de investigación" (Zúñiga \& ZúñigaHernández, 2019, p. 1029). Es por ello que se agregan como anexo dos consentimientos informados (Anexo 1 y 2) aplicados en diferentes contextos educativos, los cuales pueden ejemplificar claramente los requisitos que debe cumplir una herramienta de estas características. Vale la pena mencionar 
que se contó con la autorización de los autores de dichos consentimientos informados para que puedan ser adjuntados en este artículo.

Otros lineamientos aplicados específicamente a la investigación educativa con niños y adolescentes, es realizada por el centro de estudios MINEDUC en su documento de trabajo $N^{\circ} 14$, donde presenta las consideraciones éticas que debe tener el investigador durante el proceso de investigación. Desde esta perspectiva se ordena el proceso investigativo en cuatro etapas: diseño de investigación, implementación y el trabajo de campo, análisis de la información y devolución de los resultados y retribución de la participación.

En esta propuesta se evidencia claramente que las consideraciones éticas de la investigación en educación deben ser aplicadas durante todo el proceso investigativo. Los aspectos éticos deben ser abordados antes de comenzar el diseño de la investigación, considerado temas como la disponibilidad de la información, los beneficios o riesgos de la participación en la investigación, hasta la finalización, donde se considera la devolución de resultados y la retribución a los participantes (por ejemplo, apoyo para los traslados o la entrega de algún material como obsequio acorde a los participantes).

Es necesario subrayar que esta descripción analítica de las pautas éticas es exigua y no pretende analizar todas las particularidades contempladas en los requisitos propuestos anteriormente. Más bien, trata de ofrecer una visión global del asunto que nos permita apropiarnos de las bases explicativas fundamentales relacionada con estos códigos éticos, que proporcionan lineamientos específicosy, sin lugar a dudas, deben regir en una investigación educativa para proteger la dignidad humana, asegurar el transcurso del proceso investigativo y propiciar una adecuada socialización de los resultados alcanzados.

Por otro lado, el papel trascendental que desarrollan los comités de éticas como mecanismo evaluador, ya que estos, además de ser un filtro de extraordinario rigor, promueven una cultura ética de la investigación (Miranda, 2013). Por esa razón, dichos comités han crecido exponencialmente, puesto que evaluar un proyecto de investigación a lo largo de su desarrollo implica la participación de un equipo multidisciplinario que contribuye a develar los aspectos que pudieran volverlo deficiente desde el punto de vista ético.

A la luz de los criterios de Canario (2011), a nivel mundial los comités de ética de investigación funcionan como un espacio rector previo al inicio de las exploraciones científicas en distintas áreas del saber. De manera especial 
en la educación, su aplicación se levanta como una prueba fehaciente del uso apropiado de la ética para la comunidad que está involucrada en el desarrollo de una actividad de carácter investigativo.

A grandes rasgos, se pudiera aseverar que los mecanismos y códigos éticos abordados anteriormente nos ayudan a evaluar las propuestas de investigación dirigidas al área educativa y a velar por la seguridad y protección psicológica de los participantes en todas las etapas de la investigación.

\section{Conclusiones}

Se concluye que la investigación realizada en el campo de las ciencias de la educación en las sociedades contemporáneas es una actividad de inconmensurable valor para estimular el desarrollo humano y social. Por el hecho de involucrar seres humanos, y en algunos casos niños y adolescentes, la investigación educativa se sustenta obligatoriamente sobre un grupo de códigos éticos que vigilan la moralidad correcta de sus intenciones teóricas y metodológicas.

Respetar a cabalidad un grupo de principios éticos, nos guía en el quehacer investigativo, a alejarnos de problemáticas que limitan sustancialmente la claridad y la transparencia científica, y a su vez, nos impulsa a desarrollar prácticas que contribuyen a elevar la autonomía y aumentar los beneficios y a minimizar los riesgos potenciales a los participantes y a las agencias de socialización involucradas. Vale la pena mencionar que el cumplimiento de estos principios éticos involucra tanto a los responsables del estudio como a la comunidad educativa en sentido general.

Evaluar éticamente a través de un comité el diseño de la investigación, la selección de los grupos participantes, los procedimientos de búsqueda y análisis de la información y las vías para socializar los resultados obtenidos y la forma de retribución, constituye una tarea transcendental para dar testimonio de su mérito científico y aceptabilidad ética en los tiempos que corren. 


\section{REFERENCIAS BIBLIOGRÁFICAS}

Anderson, M., \& Steneck, H. (2011). The problem of plagiarism. Urologic Oncology: Seminars and Original Investigations, 29(1), 90-94. doi:10.1016/j. urolonc.2010.09.013

Badilla, L. (2006). Fundamentos del paradigma cualitativo en la investigación educativa. Revista de Ciencias del Ejercicio y la Salud, 4(1), 105107. Recuperado de https://revistas.ucr.ac.cr/index.php/pem/article/ view/417/402

Barrio, M., \& Simón, P. (2006). Problemas éticos de la investigación cualitativa. Med Clin (Barc). Recuperado de http://www.elsevier.es/sites/default/ files/elsevier/pdf/2 /2v126n11a13086126pdf001.pdf

Birbeck, D., \& Drummond, M. (2009). Research with young children: Contemplating methods and ethics. The Journal of Educational Enquiry, 7(2), 211-214. Recuperado de https://ojs.unisa.edu.au/index.php/EDEQ/article/view/487

Buendía, L., \& Berrocal, E. (2001). La Ética de la Investigación Educativa. España: Editorial Universidad de Huelva.

Canario, J. (2011). Comités de ética de investigación en República Dominicana: Un análisis desde las pautas éticas internacionales para la investigación biomédica con seres humanos. Acta bioethica, 17(2), 257-264. DOI: https://dx.doi.org/10.4067/S1726-569X2011000200012

Comisión Nacional de Investigación Científica y Tecnológica. (2008). Bioética en Investigación en Ciencias Sociales. 3er Taller organizado por el Comité Asesor de Bioética de FONDECYT-CONICYT. Santiago: Comisión Nacional de Investigación Científica y Tecnológica. Recuperado de https://www.conicyt.cl/fondecyt/files/2012/10/Libro-3-Bio\%C3\%A9ticaen-investigaci\%C3\%B3n-en-ciencias-sociales.pdf

Domínguez, C., \& Macías, R. (2004). El que no transa no avanza: la ciencia mexicana en el espejo, en M. Aluja \& A. Birke (coords.). El papel de la ética en la investigación científica y la educación superior (México, Fondo de Cultura Económica, 2a Ed.) pp. 219-243. 
Emanuel, E. (1999). ¿Qué hace que la investigación clínica sea ética? Siete requisitos éticos. Investigación en sujetos humanos: experiencia internacional. Santiago de Chile: Programa Regional de Bioética OPS/OMS, 33-46. Recuperado de https://www.uss. cl/wp-content/uploads/2014/12/10.-REQUISITOS-ETICOS-ENINVESTIGACIO\%CC\%81N.pdf

González, O., González, M., \& Ruiz, J. (2012). Consideraciones éticas en la investigación pedagógica: una aproximación necesaria. Edumecentro, 4(1), 1-5. Recuperado de http://scielo.sld.cu/scielo.php?script=sci_ arttext\&pid=S2077-28742012000100001\&lng=es\&tlng=es

Hammersley, M., \& Traianou, A. (2012). Ethics and educational research. London: British Educational Research Association.

Manterola, C., \& Otzen, T. (2013). Porqué investigar y cómo conducir una investigación. International Journal of Morphology, 37(4), 1498-1504. DOI: https://dx.doi.org/10.4067/s0717-95022013000400056

Ministerio de Educación, Centro de Estudios (2018). Marcos éticos para la investigación en educación con población infantil y juvenil: Hacia una propuesta de orientaciones. Documento de trabajo No 74. Santiago, Chile.

Miranda, A. (2013). Plagio y ética de la investigación científica. Revista chilena de derecho, 40(2), 711-726. DOI: https://dx.doi.org/10.4067/5071834372013000200016

Moreno, T. (2011). Consideraciones éticas en la evaluación educativa. REICE, 9(2), 130-144. Recuperado de http://scielo.sld.cu/scielo.php?script=sci_ arttext\&pid=S2077-28742012000100001\&lng=es\&tlng=es

Organización Panamericana de la Salud y Consejo de Organizaciones Internacionales de las Ciencias Médicas (2016). Pautas éticas internacionales para la investigación relacionada con la salud con seres humanos (4a Ed.). Ginebra: Consejo de Organizaciones Internacionales de las Ciencias Médicas (ClOMS).

Palamidessi, M., Gorostiaga, J., \& Suasnábar, C. (2014). El desarrollo de la investigación educativa y sus vinculaciones con el gobierno de la educación en América Latina. Perfiles educativos, 36(143), 49-66. Recuperado de http://www.scielo.org.mx/scielo.php?script=sci_arttext\&pid=S018526982014000100004\&lng=es\&tlng=es 
Rodríguez, E. (2004). Comités de evaluación ética y científica para la investigación en seres humanos y las pautas CIOMS 2002. Acta bioethica, 10(1), 37-48. DOI: http://dx.doi.org/10.4067/\$1726-569X2004000100005

Sañudo, L. (2006). La ética en la investigación educativa. Hallazgos, (6), 83-98. Recuperado de https://www.redalyc.org/articulo. oa?id=4138/413835165006

Sikes, P., Nixon, J., \& Carr, W. (2003). The moral foundations of educational research: knowledge, inquiry and values. UK: McGraw-Hill Education.

Zúñiga, C, \& Zúñiga-Hernández, J. (2019). Excepciones al uso del consentimiento informado en investigación: ¿cuándo es esto posible en Chile? Revista médica de Chile, 147(8), 1029-1035. DOI: https://dx.doi. org/10.4067/S0034-98872019000801029 


\section{ANEXO 1}

\section{CONSENTIMIENTO INFORMADO}

\section{Estimado Participante:}

La Universidad se encuentra realizando

una investigación titulada " ", desarrollada por en el marco de su Proyecto de Tesis de Magíster. El presente documento tiene como finalidad hacerle conocer los detalles del estudio y solicitarle su consentimiento informado para participar en él.

\section{Objetivo de la investigación}

El objetivo de esta investigación es:

\section{Breve descripción del proyecto}

Las políticas del paradigma de la educación inclusiva buscan asegurar las bases sociales e igualitarias para promover y potenciar una obra educativa más inclusiva que contemple las potencialidades, los intereses y las motivaciones fundamentales de todos sus educandos, substancialmente de aquellos que poseen necesidades educativas especiales. En este sentido, las políticas educativas signadas por la inclusión presentan serios desafíos a los educadores como ejes rectores del proceso de enseñanza-aprendizaje. Entonces, el entendimiento sobre cómo los educadores interactúan con estos educandos es fundamental para la creación de mejoras que aseguren la igualdad de oportunidades en el sistema escolar. Conforme a esto, el presente estudio se propone explorar la preparación de los maestros como un paso sustancial para producir mejoras en el proceso de inclusión educativa.

\section{Metodología}

El presente proyecto consta de dos momentos claves en la etapa diagnóstica: en primer lugar, con la intención de recabar información sobre las fortalezas 
y las debilidades de la actuación pedagógica de los maestros para estimular la socialización de los educandos con Espectro Autista, se observarán clases prácticas y talleres, por ser formas de organización en las que los maestros demuestran sus conocimientos y habilidades; en segundo lugar, se realizarán encuestas con el fin de develar la preparación de los maestros para potenciar la socialización de los educandos con esta condición del neurodesarrollo.

\section{Su participación en el estudio}

Es necesario apuntar, que su participación en este estudio es de carácter libre y voluntario, pudiendo solicitar ser excluido de esta investigación y que sus intervenciones no sean consideradas en esta investigación sin justificación previa ni perjuicio para usted.

Si usted participa en esta investigación lo hace bajo su expreso consentimiento informado que firma y autoriza.

\section{Confidencialidad}

La información que usted proporcione en el transcurso del estudio quedará guardada dentro de una carpeta personal del investigador responsable, y será sometida a análisis en total confidencialidad. Su identidad será resguardada por las siguientes medidas:

1. Solo el investigador responsable y su directora de tesis tendrán acceso a los datos proporcionados en los instrumentos aplicados.

2. Las encuestas recibirán un código por cada participante, que solo conocerá el investigador responsable de este estudio y su directora de tesis y, serán también realizadas en un ambiente favorable que estimule la comunicación, la confianza y el anonimato elegido por el participante.

Los resultados se utilizarán con fines científicos y se promulgarán en revistas, ponencias, congresos y otros espacios encargados de socializar el conocimiento relacionado con el ámbito educativo. En todos los casos, el Investigador Responsable asume un compromiso de confidencialidad para resguardar la identidad de todos los involucrados en este estudio. 


\section{Beneficios}

Este estudio no tiene beneficios directos para usted. En este sentido, producto de su participación no se generan incentivos económicos ni de ningún tipo. Cabe destacar también que su participación en este estudio tampoco tiene asociado ningún tipo de costo para usted, siendo el investigador responsable quien se acerca al lugar donde usted estime conveniente para la realización de la encuesta.

En tal sentido creemos que la investigación produce más bien beneficios indirectos en sus participantes puesto que les permitirá reflexionar y quizás comprender de forma holística aspectos importantes de la actuación pedagógica de los maestros para enfrentar los embates de la inclusión de educandos con Trastornos del Espectro Autista en las escuelas de enseñanza general.

\section{Riesgos o molestias asociadas a la participación}

Si se generara alguna controversia o molestia producto de alguna pregunta o reflexión durante su participación en la encuesta, el investigador responsable procurará contener emocionalmente y brindar la asistencia requerida al participante.

\section{Almacenamiento y resguardo de la información}

Toda la información que se recabe de esta investigación estará siempre resguardada y al cuidado del Investigador Responsable, quien destinará un lugar intimo (Armario ubicado en el cuarto de estudio) al interior de su casa donde se guardará todo documento de esta investigación. La llave de este lugar estará únicamente en manos del investigador responsable para evitar a toda costa su conocimiento. Todo material electrónico será debidamente almacenado y respaldado bajo una contraseña en el computador personal del Investigador Responsable.

Tanto este consentimiento, como los documentos impresos y electrónicos que se generen y sean necesarios utilizar se almacenarán por cinco años a contar del término del estudio, una vez finalizado el plazo se procederá a su eliminación de forma reservada. 


\section{Acceso a los resultados de la investigación}

Los participantes podrán consultar la información que ha generado en cualquier momento durante la ejecución del proyecto previa solicitud al investigador responsable del estudio, quien se compromete a brindar cooperación y proponer vías para tal acceso.

\section{Compromiso}

Por su aceptación los participantes se comprometen a:

1. Proveer información real en cada instancia que me sea solicitada y responder de acuerdo a mis concepciones, conocimientos y experiencias así también a utilizar mi lenguaje habitual al escribir, responder, actuar o reflexionar.

\section{Contacto}

Si usted tiene preguntas acerca de sus derechos como participante de este estudio, reclamos o dudas acerca de esta investigación, por favor contáctese con la Investigador Responsable, correo electróni$\mathrm{co}$ o con el presidente del Comité de Ética Científico de la al correo electrónico

Declaro conocer los términos de este consentimiento informado, los objetivos de la investigación, las formas de participación, de los costos y riesgos implicados, y del acceso a la información y resguardo de información que sea producida en el estudio. Reconozco que la información que provea en el curso de esta investigación es estrictamente confidencial y anónima. Además, esta será usada solo con fines de difusión científica.

He sido informado(a) de que puedo hacer preguntas sobre el proyecto en cualquier momento y que puedo retirarme del mismo cuando así lo decida, sin tener que dar explicaciones ni sufrir consecuencia alguna por tal decisión. 
Hernández, O., Antonio, A., González, D., \& Contreras, M. (2020). Consideraciones esenciales sobre el tema ético en la investigación educativa. UCMaule, 58, enero-junio, 141-164. DOI: http://doi.org/10.29035/ucmaule.58.141

Nombre completo del participante:

Correo electrónico:

Firma

Investigador(a) Responsable 


\section{ANEXO 2}

\section{CONSENTIMIENTO INFORMADO}

\section{Estimado Participante:}

La Universidad a través del programa de se encuentra realizando una investigación titulada desarrollada por en el marco de su proyecto de tesis. El presente documento tiene como finalidad hacerle conocer los detalles del estudio y solicitarle su consentimiento informado para participar en él.

\section{Objetivo de la investigación}

El objetivo de la investigación es comprender el estado actual de preparación de los profesores de enseñanza primaria respecto a la atención a la diversidad en sala de clase en el municipio de

\section{Breve descripción del proyecto}

El tema de inclusión educativa por medio de la atención a la diversidad sigue siendo discutido hoy día en cómo se entiende y como se reflecte en la práctica educativa, cómo le conciben los investigadores de la comunidad científico-académico y cómo le entienden las entidades a nivel de políticas gubernamentales de forma general y educativas de forma específica. Como refieren Sáinz y Medina (2013) la evolución educativa humana, como pocas, es una historia de superación, de lucha por la equidad y de defensa de los derechos humanos. En , de modo general el tema de atención a la diversidad carece de reconocimiento en todos sus niveles, por eso, entendemos que es de suma importancia tomar iniciativas de indagar sobre el tema desde la perspectiva del estado actual de preparación de los profesores frente a los desafíos de la inclusión educativa. Además de su objetivo general, la investigación permitirá a los agentes de la educación reconocer la gran necesidad de tener en cuenta las políticas y prácticas educativas inclusivas. 


\section{Metodología}

El presente proyecto consta de dos etapas, se realizarán entrevistas semiestructuradas y reunión de grupo focal. Para los participantes la entrevista, será de modo individual y, a la otra será una entrevista grupal. Las clases de preguntas en las entrevistas y en el grupo de enfoque, serán principalmente de opinión, de percepción, de expresión de sentimientos y de conocimientos. El local y la hora de la para los encuentros serán de consenso en respecto a tu tiempo disponible.

\section{Su participación en el estudio}

Sepa que su participación en este estudio es de carácter libre y voluntario, aunque usted acepte participar en este estudio, usted tiene derecho a abandonar su participación en cualquier momento de la investigación y que sus intervenciones no sean consideradas en esta investigación sin justificación previa ni perjuicio para usted, sin temor a ser penalizado de alguna manera.

Si usted participa en esta investigación lo hace bajo su expreso consentimiento informado que firma y autoriza.

\section{Confidencialidad}

Su participación en esta investigación es completamente anónima y confidencial.

La confidencialidad de su identidad será resguardada por la siguiente medida:

Las entrevistas recibirán un código por cada participante, que solo conoceré yo como investigador responsable de este estudio y serán realizadas en un ambiente propicio que estimule la comunicación y el anonimato elegido por el participante.

En el análisis general de los datos se utilizará una estructura de códigos para identificar la información que surja, su pertenencia al instrumento y el momento en que se realizó. Dadas las características del estudio los datos serán usados solamente en instancias académicas de investigación y aquellas propias de la divulgación investigativa. 
En la presentación de resultados no se mencionarán vuestros nombres y se reservará todo posible indicio que permita una identificación posible como lugares, instituciones, guardias, etc.

Con esto, yo asumo ante ustedes y el comité de ética de Universidad el compromiso de confidencialidad para resguardar identidad de todos los involucrados en este estudio.

\section{Beneficios}

Usted puede o no beneficiarse directamente por participar en este estudio. En este sentido, producto de su participación no se generan incentivos económicos ni de ningún tipo de costo para usted. Así siendo, yo tengo la responsabilidad de acercarme al lugar donde usted estime conveniente para la realización de la entrevista. En caso de que las circunstancias obliguen una dislocación en colectivo hasta el local acordado para la entrevista, yo aseguro el costo.

En tal sentido creemos que la investigación produce más bien beneficios indirectos a ustedes puesto que les permitirá reflexionar y quizás comprender de forma crítica el desafío de la inclusión educativa a través de prácticas educativas que atienden a la diversidad en sala de aula.

\section{Riesgos o molestias asociadas a la participación}

Al tomar parte en este estudio usted puede estar expuesto a los siguientes riesgos: incomodad al contestar preguntas sensibles, potencial pérdida de confidencialidad, o cualquier otra molestia que venga a ocurrir durante la entrevista. En caso de que se sientas molesto o incomodo por alguna pregunta que consideras sensible, lo agradezco que me hables.

\section{Almacenamiento y resguardo de la información}

Toda la información que se recabe de esta investigación estará siempre resguardada y a mi cuidado. Las entrevistas, además de las transcripciones asociadas, serán solo realizadas por mí. Todo material electrónico será debidamente almacenado y respaldado en los equipos computacionales con contraseña de la investigadora. 
Tanto este consentimiento, como los documentos impresos que se generen y sean necesarios utilizar se almacenarán por cinco años a contar del término del estudio, una vez finalizado el plazo se procederá a su eliminación de forma reservada.

\section{Acceso a los resultados de la investigación}

Ustedes podrán consultar la información que he generado en cualquier momento durante la ejecución del proyecto ante previa solicitud, yo me comprometo a brindar cooperación y proponer vías para tal acceso.

Asimismo, me compromete con cada uno de ustedes participantes a enviar el informe de investigación que se genere al final del estudio a los correos electrónicos respectivos, así también copia de los artículos científicos que pudieran resultar del estudio.

\section{Compromiso}

Me comprometo en Proveer información real en cada instancia que me sea solicitada y responder de acuerdo a mis concepciones, conocimientos y experiencias así también a utilizar mi lenguaje habitual al escribir, responder o reflexionar.

\section{Contacto}

Si usted tiene preguntas acerca de sus derechos como participante de este estudio, reclamos o dudas acerca de esta investigación, por favor contáctese con la Investigador Responsable, correo electrónico o con el presidente del Comité de Ética Científico de la Universidad al correo electrónico

Yo acepto participar voluntaria y anónimamente en el Protocolo de Investigación (Análisis del estado actual de preparación de los profesores de enseñanza primaria respecto a la atención a la diversidad en sala de clase en el municipio de programa de Magíster dictado por la facultad de Educación dirigido por la Prof. Guía 
Declaro haber sido informado/a de los objetivos y procedimientos del estudio y del tipo de participación. En relación a ello, acepto responder a una entrevista Individual/Grupal con recurso a grabación sobre el estado de preparación docente para la atención a la diversidad en sala de clase.

Declaro haber sido informado/a que mi participación no involucra ningún daño o peligro para mi salud física o mental, que es voluntaria y que puedo negarme a participar o dejar de participar en cualquier momento sin dar explicaciones o recibir sanción alguna.

Declaro saber que la información entregada será confidencial y anónima. Entiendo que la información será analizada por el investigador en forma individual o con participación de su tutora y que no se podrán identificar las respuestas y opiniones de modo personal. La información que se obtenga será guardada por el investigador responsable en dependencias de la Universidad y será utilizada sólo para fines de difusión científica.

Nombre completo del participante:

Correo electrónico:

Firma

Investigador(a) Responsable 